\title{
Integrated Business Intelligent System for E-Health: A Case for Dermatology Diseases
}

\author{
Faiza Abdulghani Al-Tajem Al-Ghamdi ${ }^{1}$, Abdullah S. Al-Malaise Al-Ghamdi ${ }^{2}$ \\ ${ }^{1}$ Dermatology Consultant, King Abdulaziz Medical City, Jeddah, KSA; ${ }^{2}$ Chairman, Information Systems Department, Faculty of \\ Computing and Information Technology, King Abdulaziz University, Jeddah, KSA. \\ Email: ghamdifa02@ngha.med.sa, aalmalaise@kau.edu.sa
}

Received December $24^{\text {th }}$, 2013; revised January $18^{\text {th }}, 2014$; accepted January $25^{\text {th }}, 2014$

Copyright (C) 2014 Faiza Abdulghani Al-Tajem Al-Ghamdi, Abdullah S. Al-Malaise Al-Ghamdi. This is an open access article distributed under the Creative Commons Attribution License, which permits unrestricted use, distribution, and reproduction in any medium, provided the original work is properly cited. In accordance of the Creative Commons Attribution License all Copyrights (C) 2014 are reserved for SCIRP and the owner of the intellectual property Faiza Abdulghani Al-Tajem Al-Ghamdi, Abdullah S. Al-Malaise Al-Ghamdi. All Copyright (C) 2014 are guarded by law and by SCIRP as a guardian.

\section{ABSTRACT}

In the current era, information technology has boosted every field of life either business industry or healthcare to integrate the internal processes of it. Due to the demand of managing huge data related to these fields numerous information systems play different roles in making the organizational processes robust and up to date. This paper discusses the integrated business intelligence implication specifically for healthcare to provide the fast and precise information on time. Therefore, this paper discusses the idea of building intelligent system based on Enterprise Resource Planning (ERP) databases using exclusively for dermatology diseases by applying data mining techniques. Firstly, classification mining has been applied for categorization data based on patient's record. Then rules and patterns generated from the categorized data related to dermatology diseases, symptoms and treatments. The proposed system will retrieve the corresponding information related to the given symptoms along with medication and complete treatment. This research aims to integrate different ERP processes with centralized ERP database to provide business intelligence effectively for the dermatologists. The paper has provided with the comprehensive conceptual framework and each step has been discussed in detail.

\section{KEYWORDS}

Dermatology; Alopecia; Business Intelligence; Data Mining

\section{Introduction}

Appropriate use of IT systems practice is the major concern of health practitioner to have an efficient management information system for effective communication, collaboration, monitoring and decision making [1,2]. Despite of the fact Health Information Systems (HIS) is the essential concern of complete practice for patients [3]. Moreover, through HIS the patients are even able to access their online record and can easily update their record. $[1,4]$ pointed out that enough budget is spent on managing of information generated from the health and make sure that the way of information managing is of high quality and with secure services. [5] has described based on [6] that information and communication technology (ICT) become very essential in healthcare like other business sectors such as baking, airlines, and manufacturing sectors. Further it is stated that, health care in the current era is 10 years back from other industries [5,6]. Computer Information systems are the essential and integral part of healthcare centers to have a computer based solution regarding the patients in hospitals. Therefore, [5] based on [7] has described that hospital information is an integrated organizational pattern for effective information sharing and ICT based solutions are provided to the healthcare centers. Data quality, data security, data privacy and data validity $[4,8]$ are the core responsibilities of every business sector to be safe and confidential in their data privacy which can be stored, validated and maintained in the hospital databases through information systems. Whenever needed, it will be retrieved through the useful interface used in the current information system for their specific concerns. 
In this paper, we will discuss the steps of building an integrated business intelligent system for healthcare information system using ERP processes and databases. These databases will further be used as a historical data for rules and patterns generation using data mining. The case selected for this paper is dermatology diseases and all related processes which will be stored in the centralized ERP databases and dermatologist communication interface will be used as a communication way between the user and the database.

\section{E-Health}

E-health is the use of technology for improving the healthcare services by using internet and other network supported media to provide the fast and easy access of health related services [9]. Similarly [10] argued that e-health is an emergent section of medical informatics used to enhance drastically the performance in term of quality access and contribution of healthcare. Healthcare provider both private and public sectors are investing an extraordinary on e-health for the improvement of patient outcomes with effective e-health services and support with low budget. Further emphasized that for achieving this vital strategy some e-health solutions will be implemented such as health care management, telemedicine system, electronic prescribing, and HIS [8]. However, e-health not only uses to manage the socio technical style of design and development systems but also reengineer the healthcare processes of e-health [9]. The sharing e-health intellectual property is a significant initiative from the developed countries to the developing countries by providing access to the e-health credential. Despite of all these, there is still several limitation of e-health in which the clinical data is not sharing explicitly and the paper based work is time consuming to maintain the patient records like medical files updates and etc $[11,12]$.

\section{Dermatology as a Case}

In this paper as we discussed in the previous sections, e-healthcare is the major concern of health organizations. Practitioners and health personnel are tremendously using the online resources to facilitate their patients with expert opinions and consultations. Dermatology is that branch of medicines which deal with the patients having any kind of diseases related to skin, hair and nail [13]. Dermatology diseases detection is a challenging task for the doctors and practitioners because the diseases of the same categories have very close features such as erythema and scaling with very minor differences [14]. Further described that there are six more groups in the same type which have similar symptoms such as; psoriasis, seboreic dermatitis, lichen planus, pityriasisrosea, chronic dermatitis and pityriasisrubrapilaris. These all has been observed in the outpatient departments [14-16]. Another hurdle during diagnosing for the doctors and practitioners that some diseases may show the features of another disease in the beginning stages and may have different features in the next stage of the same disease which is vague for the doctors to diagnose it [17]. Dermatology deal with disease such as skin, hair and nail as shown in the Figure 1.

The morphology of skin is a complex scale geometry with multi layers, air skin is oily and layers with different types of cells in epidermis, and dermis and corneum as shown in Figure 2 [19]. Eczema is a chronic skin disease in which the skin turn to reddened, itchy, crack and dry are the most common symptoms [20]. The snapshot of eczema is shown in Figure 2.

Another example to be discussed is Alopecia which is one of the diseases comes under the group of dermatology. It is a disease where a patient loss his/her hair very frequently as shown in Figure 3. Broadly it can destruction of the hair follicle, while in noncicatricial alopecia hair loss without permanent destruction of the hair follicle. Both of the type further divide in many sub-kind; cicatricial and noncicatricial alopecia. In cicatricial alopecia hair loss with permanent kind of alopecia such as; hair shaft disorder, unusual characteristics, diffusion and patterned hair loss. In any ways hair loss is frequent and sometime permanently loss [22].

\section{Teledermatology}

The recent rapidly advancement in the field of communication technology in which the equipment cost is decreasing similarly the teledermatology is growing its effect in e-healthcare [23]. Telemedicine is a way that provides online healthcare and expertise of care to the pa-

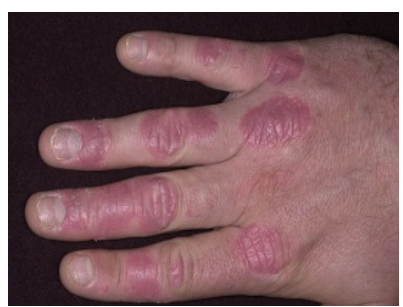

(a)

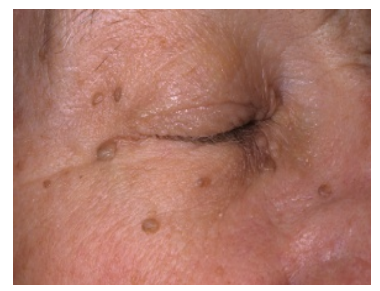

(c)

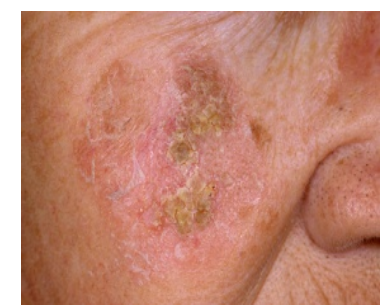

(b)

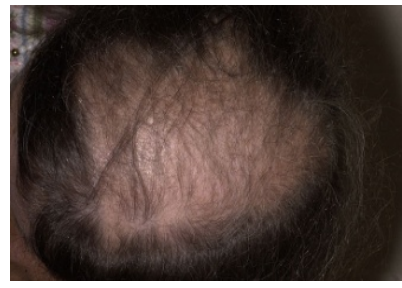

(d)
Figure 1. Dermatology diseases snapshots from the source [18]. 


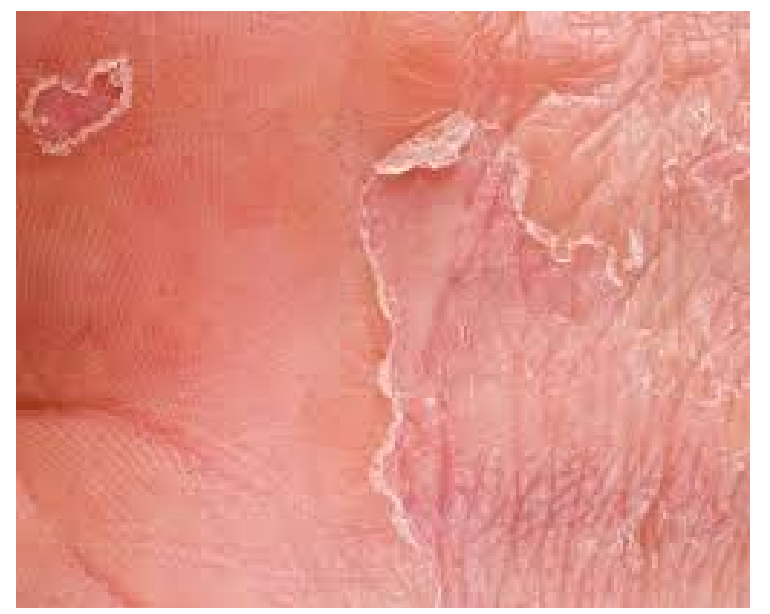

Figure 2. Eczema snapshot source [21].

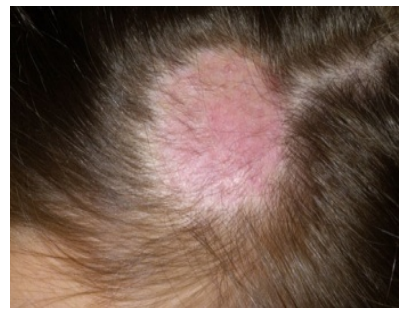

(a)

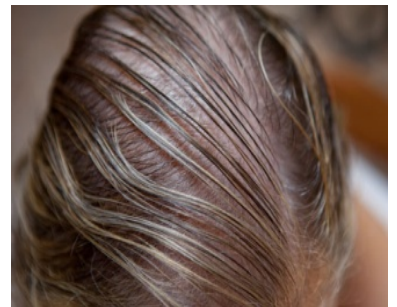

(b)

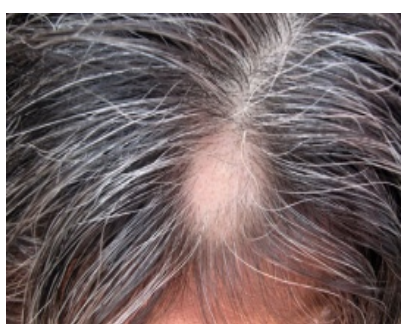

(c)

Figure 3. Alopecia-Hair loss [29].

tients by using the communication technology [24]. Telemedicine means medicine at a distance [25] and similarly telemedicine has defined very clearly by the American telemedicine association, the exchange of medical information from one site to another through online resources such as internet for patient consultation [26,27]. The main approaches used in teledermatology are store and forward technology which is time and location independent while live interactive technology needs time and location both such as video conferencing [28].

Moreover, the main theme of telemedicine is to improve the processes to the best in healthcare and minimize the cost by giving the patients with online access to medical resources [25]. Further stated that telemedicine is the opportunity of remote access to medical care as well as exchange of valuable medical information [25].

In this paper we have taken dermatology diseases as a case using a conceptual framework for stored datain in- tegrated centralized ERP database for extraction of a business intelligence system for e-health. The detail discussion of methodology and proposed framework has been described in the following section.

\section{Methodology}

As per the above discussion it is evident from the literature and different online healthcare centers resources that dermatology is dealing with several diseases and high number of patients around the globe [18,20,21,23,27,29]. In this paper we have built a conceptual framework for business intelligence system to making it fast and efficient in response of asked queries from the system. The data related to each and every dermatology disease, symptoms, treatments, precautions, and patient history will be saving in ERP centralized database after processing. Upon the user (Doctor, practitioner, dermatologist etc) queries for the entered symptoms through dermatologist communication interface will retrieve all the related diseases and treatments for diagnosing of a concern patient through intelligence system.

1) Healthcare ERP Processes

In the proposed framework, an ERP systems consist of four different type of databases such as; dermatology patient record, dermatology symptoms, dermatology disease, and dermatology medications which is further collectively stored in hospital centralized database. Each and every database deal with the corresponding data and ERP processes related with dermatology patients. The centralized database will further connected with healthcare intelligence process phase.

2) Healthcare Intelligent Process

The first step in this phase is to analyze the data from hospital centralized database for implementation of data mining techniques such as; classification and association mining as shown in Figure 4. To categories the diseases in many classes such as; Skin, Hair, and Nailand subclasses such as; alopecia, eczema, cicatricial and noncicatricial of dermatology cases using classification mining.

Furthermore, association mining will use for mapping and association between different classes, subclasses, their symptoms and treatments. Moreover, rules and patterns can be extracted from the categorized data which has been described in discussion section.

\section{Discussion on Presented Framework}

The framework presented in Figure 4, is the conceptual illustration of the proposed e-healthcare business intelligent system specifically designed for dermatology cases. The proposed framework will be able to handle all such queries in the same way as ask from the human expert (dermatologist). Regardless of expert opinion, the system 


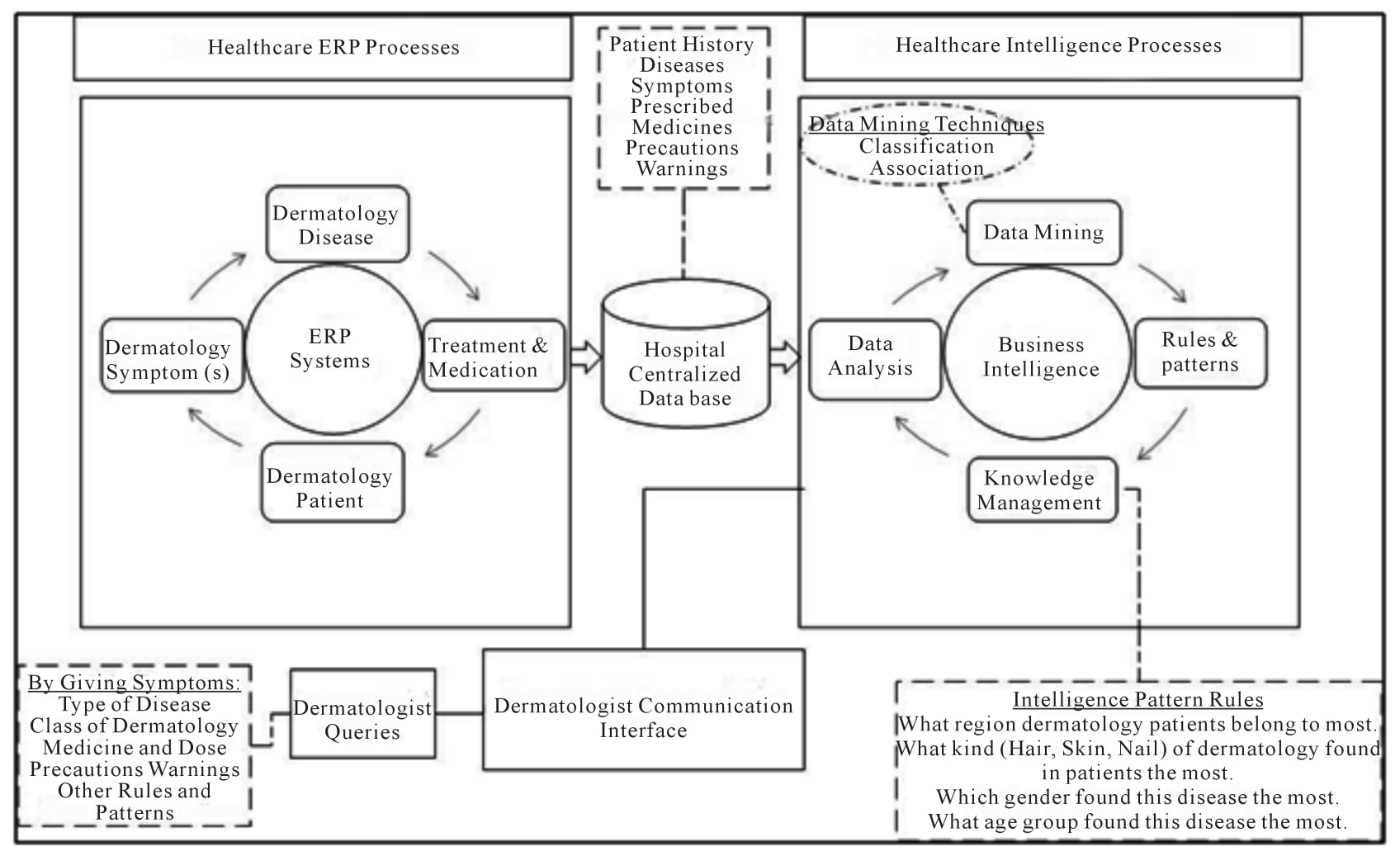

Figure 4. Proposed framework-Integrated business intelligent system of e-health for dermatology cases.

will provide the results with less time and in more efficient way without connecting to the main hospital database. As normally, the healthcare intelligent processes will work online/offline to process newly entered data and will rapidly create rules and patterns which will be then forwarded to knowledge management for fast response to the dermatologist based on the asked queries. The system is depending on the knowledge management which is interrelated with the data mining directly as well as with hospital centralized database indirectly.

The main process in the proposed framework initiates from the ERP database, which is main source of data having several ERP processes running in the healthcare ERP systems such as; dermatology symptoms, disease patients and medications. Several kinds of data related with diseases and patients, processed under the supervision of doctors, specialists and consultants. Moreover, out of this huge amount of data some attributes has been selected for the data mining implementation such as; patient history, diseases, symptoms, prescribed medicines, precautions and warnings. Those attributes will provide the help in comprehensive analysis through classification and association mining, which will lead ultimately into the process of creation rules and patterns.

Healthcare intelligence processes as shown in Figure 4 is the major step established for discovery of new and unseen data. In this phase, knowledge discovery will be applied on patient data selected from hospital centralized
ERP database. First of all classification mining will be applied for transforming all data into different categories of dermatology cases. A dermatology patient may have different kind of symptoms and diseases as discussed in the literature, such as hair, skin and nail. The data need to be classified according to subclasses of dermatology before implementation of association mining.

In this discussion, Alopecia disease has been taken as an example to present the information flow in the model. Alopecia is the common disease for hair loss which comes under the group of dermatology diseases. [22] described in details about Alopecia (hair loss) disease which can be occur due to many reasons such as; hair shaft disorder, and other kind of hair loss. Disorder and unusual characteristics may create the disease alopecia. Alopecia may have other types like cicatricial (scarring) and noncicatricial alopecia. Diffusion and patterned hair loss are two other subkinds of cicatricail and noncicatricial alopecia. In any kind of discussed cases the hair will be permanently loss. While in patterned alopecia certain areas of the scalp are affected more than others [22]. The classification table of above discussion is shown in Table 1.

The implementation of classification will allow transforming data into categorized format which is easy to read and understand. The classification of Alopecia diseases shown in Table 1 is an example of data sets after applying classification. To provide visual understanding 
of the disease, a database may contain some figures related to the corresponding disease such as shown in Figures 5-7.

Next step in this framework is to extract relationship between the symptoms and diseases stored in ERP databases. For this, association mining will be applied on classified data to create association rules and patterns to be save in knowledge management as a knowledge. The output of association mining is to create number of standard patterns which will be directly or indirectly associated with the disease. For example, if the person having the symptoms of hair shaft disorder then it may diagnose as the Cicatricial or Noncicatrical based on other characteristics discussed in the Table 1, where both of these diseases are subclasses of Alopecia and finally alopecia is relate to dermatology, a branch of medicine. Then the patient will be forwarded or treated by dermatologist with proper medications and will suggest the required precautions which is already described in the centralized database. The database contained all diseases and its medication for healing. The discussed example will be processed through the knowledge management using a defined rules generated by association mining as follows:

If person has hair shaft disorder $\rightarrow$ Cicatricial/Noncicatrical (based on other particular characteristics) $\rightarrow$ Alopecia $\rightarrow$ Dermatology Medications.

Some other kinds of rules can be generated using the classified datasets such as; under what age the particular disease found most? What gender has particular disease most? And so on. Finally, through the dermatologist communication interface different kind of queries will deal with knowledge management. The proposed system can provide the help to the dermatologist (selected case) by providing the rules and patterns generated from the historical data. Every time the query will perform the task, new information and experienced data automatically will be stored in knowledge management for future concerns. The proposed idea discussed with the example of dermatology diseases. The same framework can be applied for other kind of diseases, subclasses, symptoms and medications.

\section{Conclusion \& Future Work}

The conclusion of this paper emphasizes the integrated business intelligent system of healthcare sectors. This paper has proposed a framework to make the search time robust for the dermatologist during diagnosing the patients diseases. The proposed framework illustrates the significance of the presented idea by using data mining techniques for categorization and association between the symptoms and diseases \& treatment. The selected case in dermatology for this paper was alopecia as discussed above in the discussion section. Therefore the classified

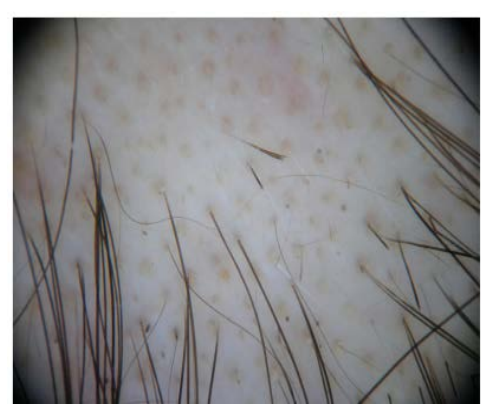

Figure 5. Alopecia areata: yellow dots and exclamation mark hairs [30].

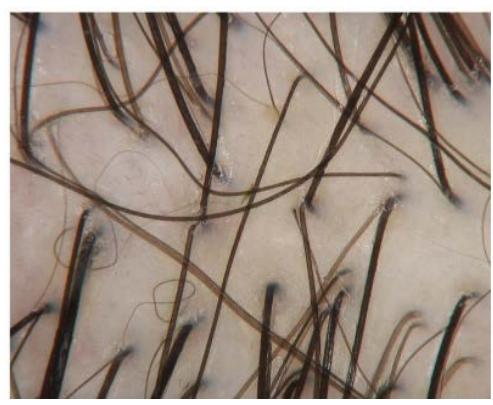

Figure 6. Androgenetic alopecia: hair diameter variability [30].

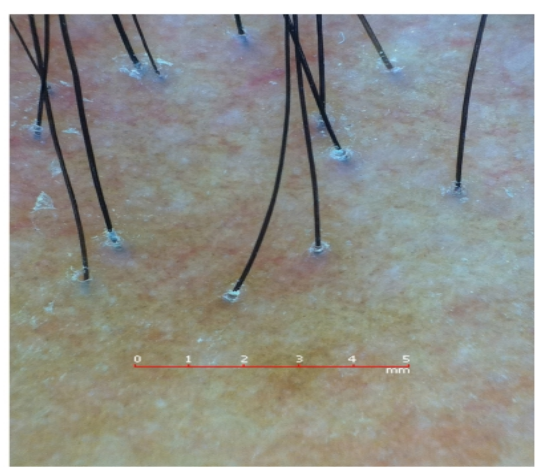

Figure 7. Lichen planopilaris: loss of follicular openings and perifollicular casts [30].

Table 1. Classification of alopecia [22].

\begin{tabular}{cccc}
\hline Main Class & Sub-Classes & Symptoms \\
\hline $\begin{array}{c}\text { Alopecia } \\
\text { (A Disease of dermatology) }\end{array}$ & Cicatricial & Hair shaft Disorder \\
& (Hair loss with permant destruction of the hair follicle) & Unusual characteristics \\
Diffusion & Noncicatricial & Prescribed by the \\
dermatologists & Patterned hair loss & \\
\hline
\end{tabular}


table using classification mining and generated rules using association mining from the ERP centralized database for alopecia disease has been presented to show the work flow of the proposed model. Moreover, in future the model will be enhanced and validated by using empirical data collected from dermatology databases. Building database with multimedia and visual support can also be enhanced with the actual idea of this paper. The model has a general concept itself, which can be applied for other kind of ERP based health system in future. The proposed system can be amended to accommodate other diseases as well. The approach can be used for online purpose by keeping the system on cloud.

\section{REFERENCES}

[1] "eHealth: The Future of Health Care, Royal College of Nursing Position Statement," The Royal College of Nursing, London, 2012.

[2] L. McQueen, H. Carter-Templeton and K. A. McCormick, “Translation of Evidence into Nursing Practice,” In: V. K. Saba and K. A. McCormick, Eds., Essentials of Nursing Informatics, 5th Edition, McGraw Hill, New York, 2011, pp. 391-410.

[3] L. A. Huryk, "Factors Influencing Nurses' Attitudes towards Healthcare Information Technology,” Journal of Nursing Management, Vol. 18, No. 5, 2010, pp. 606-612. http://dx.doi.org/10.1111/j.1365-2834.2010.01084.x

[4] "Guidance on Privacy Impact Assessment in Health and Social Care,” Health Information and Quality Authority, 2010.

[5] P. Locatelli, N. Restifo, L. Gastaldi and M. Corso, "Health Care Information Systems: Architectural Models and Governance," Innovative Information Systems Modelling and Techniques, InTech, Rijeka, 2012, pp. 71-96.

[6] W. Raghupathi, "Information Technology in Healthcare: A Review of Key Applications,” In: K. Beaver, Ed., Healthcare Information Systems, 2nd Edition, Boca Raton, 2003, pp. 9-27.

[7] M. Corso and L. Gastaldi, "Managing ICT-Driven Innovation in the Health Care Industry: Evidence from an Empirical Study in Italy," Proceedings of the 10th CINet Conference, Brisbane, 6-8 September 2009, pp. 1-14.

[8] "Information Governance. The Foundation for Effective e-Health, Accenture,” Institute of Health \& Public Service Value, 2010.

[9] P. Nykänen, "eHealth Research and Practice-Achievements and Challenges for the Future," IMMODI eHealth Forum, Vuokatti, 2011. http://www.kainuunetu.fi/UserFiles/d763e312-4069-4815 -82bd-f6886b1871ca/Web/IMMODI/IMMODI_Vuokatti _16062011_PirkkoNykanen.pdf

[10] E. Vasilyeva, M. Pechenizkiy and S. Puuronen, “Towards the Framework of Adaptive User Interfaces for eHealth,” Proceedings of the 18th IEEE Symposium on ComputerBased Medical Systems (CBMS’05), 2005, pp 139-144. http://dx.doi.org/10.1109/CBMS.2005.101
[11] P. Germanakos, C. Mourlas and G. Samaras, “A Mobile Agent Approach for Ubiquitous and Personalized eHealth Information Systems," Proceedings of the Workshop on Personalization for e-Health of the 10th International Conference on User Modeling, 2005, pp. 67-70.

[12] F. E. Sanz de León, P. Nicklin, C. Rhodes and S. Y. Kwankam, "Promoting Appropriate eHealth Technologies in the Developing World: The Sharing eHealth Intellectual Property for Development (SHIPD) Initiative of the World Health Organization," Seminar on Appropriate Healthcare Technologies for Developing Countries, 2008, pp. 1-3.

[13] Dermatology. http://en.wikipedia.org/wiki/

[14] N. Rajkumar and P. Jaganathan, “A New RBF Kernel Based Learning Method Applied to Multiclass Dermatology Diseases Classification,” Proceedings of 2013 IEEE Conference on Information and Communication Technologies (ICT 2013), 2013, pp. 551-556.

[15] G. Demiroz, H. A. Govenir and N. Ilter, "Learning Differential Diagnosis of Erythemato-Squamous Diseases Using Voting Feature Intervals," Artificial Intelligence in Medicine, Vol. 13, 1998, pp. 147-165. http://dx.doi.org/10.1016/S0933-3657(98)00028-1

[16] H. A. Govenir and N. Emeksiz, “An Expert System for the Differential Diagnosis of Erythemato-Squamous Diseases,” Expert Systems with Applications, Vol. 18, No. 1, 2000, pp. 43-49.

http://dx.doi.org/10.1016/S0957-4174(99)00049-4

[17] A. Azzini and S. Marrara, "Dermatology Disease Classification via Novel Evolutionary Artificial Neural Network," 18th International Workshop on Database and Expert Systems Applications, 2007, pp. 148-152.

[18] Dermet, 2013. http://www.dermnet.com/

[19] S. K. Madan, K. J. Dana and O. G. Cula, "Quasiconvex Alignment of Multimodal Skin Images for Quantitative Dermatology," IEEE Conference on Computer Vision and Pattern Recognition Workshops, 2009, pp. 117-124.

[20] MNT Knowledge Center, 2013. http://www.medicalnewstoday.com/articles/14417.php

[21] Medicine Net, 2013. http://www.medicinenet.com/image-collection/eczema_pi cture/picture.htm

[22] G. Eudy and A. R. Solomon, "The Histopathology of Noncicatricial Alopecia," Seminars in Cutaneous Medicine and Surgery, Vol. 25, No. 1, 2006, pp. 35-40. http://dx.doi.org/10.1016/j.sder.2006.01.005

[23] British Association of Dermatologist, Healthy Skin for All, "The Role of Teledermatology in the Delivery of Dermatology Services,” 2013. http://www.bad.org.uk/Portals/_Bad/Clinical\%20Services /BAD\%20Teledermatology\%20Position\%20Statement\% 20rev1\%20April\%202010.pdf

[24] K. Prabaharan, J. Lavanya, K. W. Goh, Y. Kim and C. B. Soh, "Distributed Architecture towards Tele-Diagnosis," Proceedings of the 1st Distributed Diagnosis and Home Healthcare (D2H2) Conference Arlington, Virginia, 2-4 April 2006.

[25] G. Romero, J. A. Garrido and M. García-Arpa, “Teleme- 
dicine and Teledermatology (I): Concepts and Applications,” 2013. http://http:://www.revespcardiol.org

[26] J. Ginger Han, B. Anderson, I. Gireada, J. Dyer and C.-R. Shyu, "e-Health Networking Applications and Services (Healthcom)," 13th IEEE International Conference on e-Health Networking, Application and Services, 2011, pp. 278-281.

[27] American Telemedicine Association, 2013. http://www.americantelemed.org

[28] C. R. Jung and J. Scharcanski, "Sharpening Dermatolog- ical Color Images in the Wavelet Domain,” IEEE Journal of Selected Topics in Signal Processing, Vol. 3, No. 1, 2009, pp. 4-13.

http://dx.doi.org/10.1109/JSTSP.2008.2011113

[29] Web MD, 2013.

http://www.webmd.com/skin-problems-and-treatments/ha ir-loss/ss/slideshow-womens-hair-loss

[30] K. A. Gordon and A. Tosti, "Alopecia: Evaluation and Treatment," Clinical, Cosmetic and Investigational Dermatology, 2011, pp. 101-106. 